\title{
Highly efficient magnetic targeting of mesenchymal stem cells in spinal cord injury
}

This article was published in the following Dove Press journal:

International Journal of Nanomedicine

13 July 2012

Number of times this article has been viewed

\section{Václav Vaněček ${ }^{1,5}$ \\ Vitalii Zablotskii ${ }^{2}$ \\ Serhiy Forostyak ${ }^{1,5}$ \\ Jiří Růžička' \\ Vít Herynek ${ }^{3}$ \\ Michal Babič ${ }^{4}$ \\ Pavla Jendelová 1,5 \\ Šárka Kubinová \\ Alexandr Dejneka ${ }^{2}$ \\ Eva Syková ${ }^{1,5}$}

'Institute of Experimental Medicine AS CR, Prague, Czech Republic; ${ }^{2}$ Institute of Physics AS CR, Prague, Czech Republic; ${ }^{3}$ MR-Unit, Institute for Clinical and Experimental Medicine, Prague, Czech Republic; ${ }^{4}$ Institute of Macromolecular Chemistry AS CR, Prague, Czech Republic; ${ }^{5}$ Department of Neuroscience, 2nd Faculty of Medicine, Charles University, Prague, Czech Republic
Correspondence: Václav Vaněček Institute of Experimental Medicine AS CR, Vídeňská 1083, 14220 Prague 4, Czech Republic

$\mathrm{Tel}+420241062717$

Fax +420 24I 062706

Email vanecek@biomed.cas.cz
Abstract: The transplantation of mesenchymal stem cells (MSC) is currently under study as a therapeutic approach for spinal cord injury, and the number of transplanted cells that reach the lesioned tissue is one of the critical parameters. In this study, intrathecally transplanted cells labeled with superparamagnetic iron oxide nanoparticles were guided by a magnetic field and successfully targeted near the lesion site in the rat spinal cord. Magnetic resonance imaging and histological analysis revealed significant differences in cell numbers and cell distribution near the lesion site under the magnet in comparison to control groups. The cell distribution correlated well with the calculated distribution of magnetic forces exerted on the transplanted cells in the subarachnoid space and lesion site. The kinetics of the cells' accumulation near the lesion site is described within the framework of a mathematical model that reveals those parameters critical for cell targeting and suggests ways to enhance the efficiency of magnetic cell delivery. In particular, we show that the targeting efficiency can be increased by using magnets that produce spatially modulated stray fields. Such magnetic systems with tunable geometric parameters may provide the additional level of control needed to enhance the efficiency of stem cell delivery in spinal cord injury.

Keywords: magnetism, mesenchymal stem cell, nanoparticle, spinal cord injury, modeling

\section{Introduction}

Spinal cord injury (SCI) is a devastating traumatic injury leading to the loss of neurons, axonal degeneration, a serious neurological deficit, and permanent invalidity. Despite extensive research, there is currently no effective therapy for SCI. ${ }^{1,2}$ The development of new methods that allow for the restoration of tissue function in the injured spinal cord represents a major challenge for regenerative medicine. One of the promising treatment approaches tested in preclinical and clinical studies is the transplantation of stem cells into the damaged spinal cord. ${ }^{3,4}$

Mesenchymal stem cells (MSC) are under intensive study as a potential therapeutic tool, particularly for the treatment of bone,${ }^{5}$ cartilage, ${ }^{6}$ cardiac $^{7}$ and neural diseases, including stroke, ${ }^{8}$ amyotrophic lateral sclerosis, ${ }^{9}$ and SCI. ${ }^{10,11}$ Numerous studies have demonstrated their benefit in promoting anatomic and functional recovery after transplantation in animal models of SCI. ${ }^{11-14}$ The mechanisms underlying the therapeutic effect of MSC are primarily the secretion of growth factors and cytokines supporting neuronal repair, ${ }^{15}$ immunomodulation, ${ }^{16}$ and antiapoptotic effects,,${ }^{17,18}$ which promote regenerative processes in the central nervous system.

Important parameters influencing the effect of cell transplantation are the number of transplanted cells, the site of transplantation, as well as the route of administration. 
Less invasive cell transplantation, but sufficient cell retention and engraftment in the tissue of interest, are essential for the effective implementation of cellular transplantation strategies in regenerative medicine. When administering stem cells intended to affect SCI, direct injection, lumbar puncture, and intravenous or intraarterial injection are the commonly used methods. Intrathecal administration via lumbar puncture has been described as being more efficient than intravenous administration and less invasive in comparison to direct injection. ${ }^{19}$ As the number of cells in the lesion is one of the critical parameters, it is important to find an efficient means of cellular delivery, ideally one that would concentrate or facilitate the homing of cells to the site of injury.

For monitoring the efficiency of cell transplantation, cellular homing or targeting, grafted cells can be labeled with superparamagnetic iron oxide nanoparticles (SPION) and detected by means of magnetic resonance imaging (MRI). ${ }^{20,21}$ In addition, cells labeled with SPION can be manipulated in a magnetic field ${ }^{22,23}$ and successfully targeted to different organs. Previous studies in this area have focused on targeting cells into the liver ${ }^{24}$ or injured brain ${ }^{25}$ or have evaluated the homing of cells into the healthy spinal cord with the use of a magnetic system based on a permanent magnet. ${ }^{26}$ However, a more detailed evaluation of such magnetic systems is often missing in the literature.

In an effort to improve the efficiency of cell transplantation into the damaged spinal cord, we evaluated both theoretically and experimentally an in vivo magnetic targeting system based on a slab-shaped commercially available permanent magnet. We propose an improved magnetic system for targeting labeled cells in a spinal cord lesion and provide a mathematical model describing such cell targeting.

\section{Materials and methods} Cell preparation

MSC were obtained from 4-week-old green fluorescent protein (GFP) transgenic Sprague Dawley rats, transgenic line code $\mathrm{TgN}$ (acro/act-EGFP) 4Osb. The animals were deeply anesthetized, the femurs and tibias were dissected and the bone marrow was plated on Petri dishes containing Dulbecco's modified Eagle's medium (PAA Laboratories GmbH, Pasching, Austria), 10\% fetal bovine serum (PAA Laboratories $\mathrm{GmbH})$, and Primocin ${ }^{\mathrm{TM}}(100 \mu \mathrm{g} / \mathrm{mL}$; Lonza Cologne AG, Koeln, Germany). Cells were allowed to adhere; nonadherent cells were removed after 48 hours by replacing the medium. Adherent cells were cultivated at $37^{\circ} \mathrm{C}$ in a humidified atmosphere containing $5 \% \mathrm{CO}_{2}$, and the medium was changed twice a week. After reaching near-confluency, the cells were harvested by a Trypsin/EDTA solution (Gibco-Invitrogen, Carlsbad, CA). After two to three passages, the cells were labeled with SPION and transplanted into animals. The cells were characterized as MSC by their spindle-shaped morphology and adherence to plastic, while their multipotency was confirmed by their differentiation into adipocytes, osteoblasts, and chondroblasts according to a standard differentiation protocol., ${ }^{9,27}$ The cells were positive for CD90 and fibronectin and negative for CD11b and CD $45 .^{20}$

\section{Cell labeling with SPION}

For cell labeling, poly-L-lysine-coated SPION were used in this study. The nanoparticles were prepared and characterized as described previously. ${ }^{28}$ Cultures of MSC were incubated with SPION $(50 \mu \mathrm{L} / 10 \mathrm{~mL}$ of culture medium, ie, $15.4 \mu \mathrm{g}$ of iron/1 mL media) 72 hours prior to experiments. After 72 hours, the nanoparticles were washed out and the labeled cells were implanted into the animals.

For in vitro experiments, SPION-labeled cells were plated in a Petri dish (100,000 cells/dish) with a permanent magnet or a nonmagnetic plastic substitute fixed on the bottom of the dish. After 48 hours, the magnet or nonmagnetic substitute was removed and the cells were fixed with paraformaldehyde. The distribution of cells in the Petri dish was observed under a fluorescence microscope.

\section{Balloon-induced spinal cord compression lesion model}

The use of animals in this study was approved by the ethics committee of the Institute of Experimental Medicine ASCR (Prague, Czech Republic). All efforts were made to minimize the number of animals used in the study. A balloon compression lesion was performed in a total of 32 male Wistar rats (280-300 g) as described by Urdzikova et al. ${ }^{11}$ Briefly, the animals were anesthetized with $2 \%$ isoflorane (Forane ${ }^{\circledR}$; Abbott Laboratories, Queenborough, UK) and shaved on the back from C7 to Th 12. Under sterile conditions, the skin was cut in the midline from Th7-Th12. The soft tissue was removed, as well as the spinous processes of vertebrae Th8-Th11. A catheter was filled with saline and connected to a Hamilton syringe. The catheter was inserted into the epidural space and advanced cranially for $1 \mathrm{~cm}$, so that the center of the balloon rested at the Th8-Th9 level of the spinal cord. The balloon was rapidly inflated with $15 \mu \mathrm{L}$ of saline for 5 minutes. The catheter was then deflated and removed. Subsequently, a small magnetic implant $(13 \times 7 \times 2 \mathrm{~mm}$, neodymium magnet with magnetic 
induction at the surface $\mathrm{B}_{\mathrm{S}}=0.35 \mathrm{~T}$ and remnant magnetic field $\mathrm{B}_{\mathrm{r}}=1.2 \mathrm{~T}$; ABC Magnet Ltd, Prague, Czech Republic) covered with a biocompatible silicone plastic coating or a non-magnetic plastic implant was inserted on the top of the vertebral column at Th8-Th9 (above the lesion site) and fixed and sutured in the muscles, so that the surface of the magnet rested approximately $4-4.5 \mathrm{~mm}$ above the lesion site (considering the thickness of the vertebral bone [lamina], the tissue below the magnet, and the magnet coating). The soft tissue and skin were sutured with unresorbable thread, and the animals were allowed to feed and drink ad libitum.

\section{Cell transplantation}

The rats were transplanted intrathecally 1 week after injury. To study the effect of magnetic targeting as a function of time, animals were sacrificed 12, 24, 72, or 168 hours after transplantation. A lumbar puncture was performed as described previously. ${ }^{29}$ The rats were anesthetized with $2 \%$ isoflorane, and the skin was cut at the L5-L6 level. For each time interval, a group of animals with a magnet $(n=4)$ and another group with a nonmagnetic substitute $(n=4)$ were injected with 0.5 million SPION-labeled MSC in $50 \mu \mathrm{L}$ of phosphate-buffered saline (PBS) using a sterile $25 \mathrm{G}$ needle. All animals were immunosuppressed daily with cyclosporine (10 mg/kg, ip, Sandimmun ${ }^{\circledR}$; Novartis, Basel, Switzerland), and bacterial infection was prevented by gentamicine (0.05 mL, im, Gentamicine Lek ${ }^{\circledR}$; Lek Pharmaceuticals, Ljublanja, Slovenia).

\section{Magnetic resonance imaging}

At the end of the experiment, the animals were intracardially perfused under deep anesthesia (pentobarbital $150 \mathrm{mg} / \mathrm{kg}$; Sigma, St Louis, MO) with 4\% paraformaldehyde in $0.1 \mathrm{M}$ PBS. The vertebrae were dissected and ex-vivo magnetic resonance images (MRI) were obtained using a 4.7 T Bruker spectrometer (Bruker BioSpin, Ettlingen, Germany) equipped with a volume resonator coil. Sagittal images were obtained using a standard T2-weighted turbo spin-echo sequence. The sequence parameters were: repetition time $(\mathrm{TR})=2000 \mathrm{~ms}$, effective echo time $(\mathrm{TE})=70 \mathrm{~ms}$, turbo factor $=16$, number of acquisitions $(\mathrm{AC})=48$, slice thickness $=0.75 \mathrm{~mm}$, $\mathrm{FOV}=5 \times 2.5 \mathrm{~cm}$ and matrix $256 \times 256$.

\section{Histology and image analysis}

Spinal cords were dissected, postfixed overnight in $4 \%$ paraformaldehyde, and transferred to $10 \%$ and $20 \%$ sucrose. After freezing, the spinal cords were cryosectioned into longitudinal sections (14 $\mu \mathrm{m}$ thickness) and counterstained with DAPI. In order to visualize the colocalization of the GFP signal with SPION labeling, iron in the serial histological sections was detected by staining with potassium ferrocyanide (Lachema, Brno, Czech Republic) to produce ferric ferrocyanide (Prussian blue) according to a standard staining protocol. To detect the possible infiltration of macrophages in the lesion, staining for CD68 (ED1; Serotec, Oxford, UK) was performed followed by counterstaining with Nuclear Fast Red (Sigma). Antigen-antibody complexes were visualized using a biotin-streptavidin detection system (LSAB2 System, HRP; DakoCytomation, Carpinteria, CA) with 3,3'-diaminobenzidine as the chromogen (Vector Laboratories, Burlingame, CA). The number of $\mathrm{GFP}^{+}$cells was quantified using a procedure described previously. ${ }^{30}$ $\mathrm{GFP}^{+}$cells containing a DAPI-positive nucleus were counted in every sixth section in an $18 \mathrm{~mm}(9 \times 2 \mathrm{~mm})$ segment with the epicenter of the lesion in the center of the segment. The total number of surviving transplanted cells was obtained by multiplying the number of cells in the counted sections by 6 to compensate for the sampling frequency. Histological and image analyses were performed using a Zeiss Axioskop Light microscope (Carl Zeiss, Rochester, NY) and image analysis software.

\section{Statistical analysis and mathematical modeling}

The statistical significance of differences in cell counts in the spinal cord lesions between the magnet and nonmagnet groups was determined using Student's $t$-test. Differences were considered statistically significant if $P<0.05$. Data are expressed as means \pm standard error of mean. In the subarachnoid space the distribution of the magnetic forces acting on the magnetically labeled cells from a permanent magnet was calculated analytically with the help of explicit expressions for the magnetic stray fields. ${ }^{31}$ The kinetics of the cells' accumulation near the lesion site are described by a mathematical model elaborated with regard to both the biological and physical factors affecting the cell distribution.

\section{Results}

\section{In vitro magnetic targeting}

In vitro studies showed that in a Petri dish, MSC labeled with SPION can be attracted by magnetic force to the region above the magnet (Figure 1A and C). In control dishes, without the influence of the magnet, the cells were uniformly distributed (Figure 1B and D). Prussian blue staining confirmed the presence of iron oxide nanoparticles in the cells. 


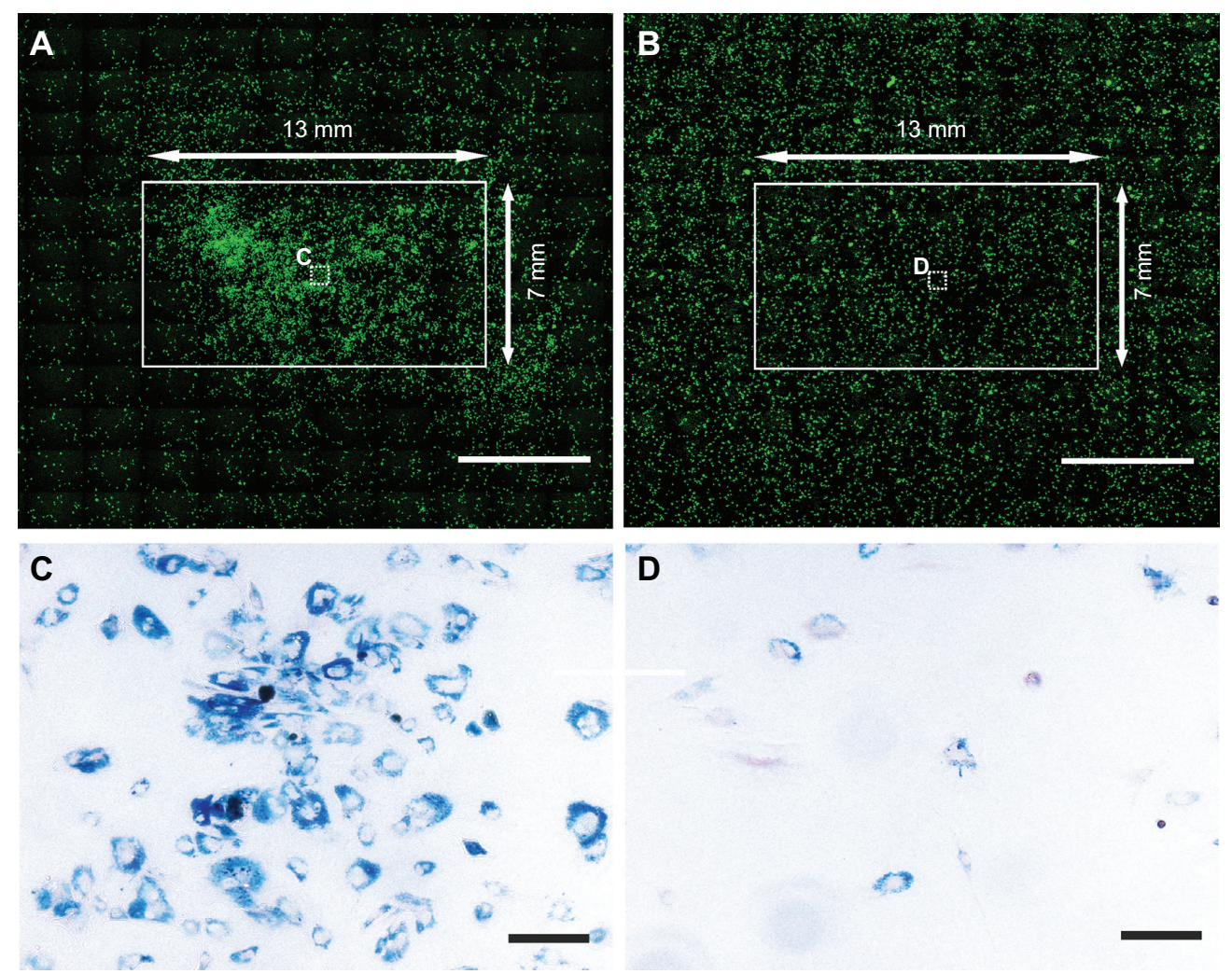

Figure I In the magnet group (A), GFP cells labeled with SPION and seeded in a Petri dish were attracted to the region above the magnet $($ I $3 \times 7 \times 2 \mathrm{~mm}$, the white solid-line rectangle depicts the magnet's edges). In the control group (B), the cells were spread uniformly throughout the dish and did not concentrate in the region above the plastic substitute. (C and $\mathbf{D})$ show enlarged views of the white boxes in the middle of the magnet (A) or nonmagnet area (B).

Notes: Cells were stained for iron to produce Prussian blue. Scale bars $=5000 \mu \mathrm{m}(\mathbf{A}$ and $\mathbf{B})$ and $50 \mu \mathrm{m}(\mathbf{C}$ and $\mathbf{D})$.

Abbreviations: GFP, green fluorescent protein; SPION, superparamagnetic iron oxide nanoparticles.

\section{Magnetic targeting in a spinal cord lesion}

Histological analysis of spinal cord sections revealed transplanted SPION-labeled $\mathrm{GFP}^{+}$cells in both the magnet and control groups. MRI scans and histological findings were similar in the magnet groups at each time point, as were the scans and findings for the control groups at each time point. In order to avoid needless duplication, Figure 2 shows representative MRI and histological images of a spinal cord in both the magnet and control groups at a single time point, 72 hours after transplantation. MRI scans showed a larger hypointense signal of SPION-labeled cells in the lesion area in the magnet group (Figure 2A) in contrast to the weak signal observed in the control group (Figure 2D). In the magnet groups, the cells were located in clusters mostly in the dorsal intrathecal space of the spinal cord below the magnetic implant. Prussian blue staining (Figure 2B) colocalized with the GFP signal (Figure 2C) in serial histological sections. The observed Prussian blue-positive cells were negative for ED1-DAB staining, thus excluding the presence of local macrophages loaded with iron (Figure $2 \mathrm{~B}$ and $\mathrm{E}$ ). In the control groups, fewer cells were identified in the lesion area (Figure 2E and F). The cell distribution in the magnet groups correlated with the magnetic forces of the magnetic implant above the lesion site (see details in the text below), which attracted and concentrated the cells near the lesion and prevented the cells from advancing more cranially (Figure 3). In the control groups, the cells were distributed more uniformly along the measured spinal cord segment. Quantitative analysis revealed that the mean total number of cells found in the measured segment in the magnet groups was the highest 12 hours after cell administration (9595 \pm 2231 cells), followed by a continual decrease after 24 hours ( $7181 \pm 939$ cells), 72 hours (3002 \pm 581 cells) and 168 hours ( $2734 \pm 174$ cells). In the controls, the number of cells 12 hours after administration ( $3538 \pm 625$ cells) was significantly lower in comparison to the magnet group $(P<0.05)$. The highest number was found after 24 hours (6894 \pm 1520 cells), followed by a decrease after 72 hours (1837 \pm 600 cells) and 168 hours (720 \pm 138 cells); the number of cells observed at 168 hours was significantly lower $(P<0.05)$ in comparison with the cell number found at the same time point in the magnet group. 


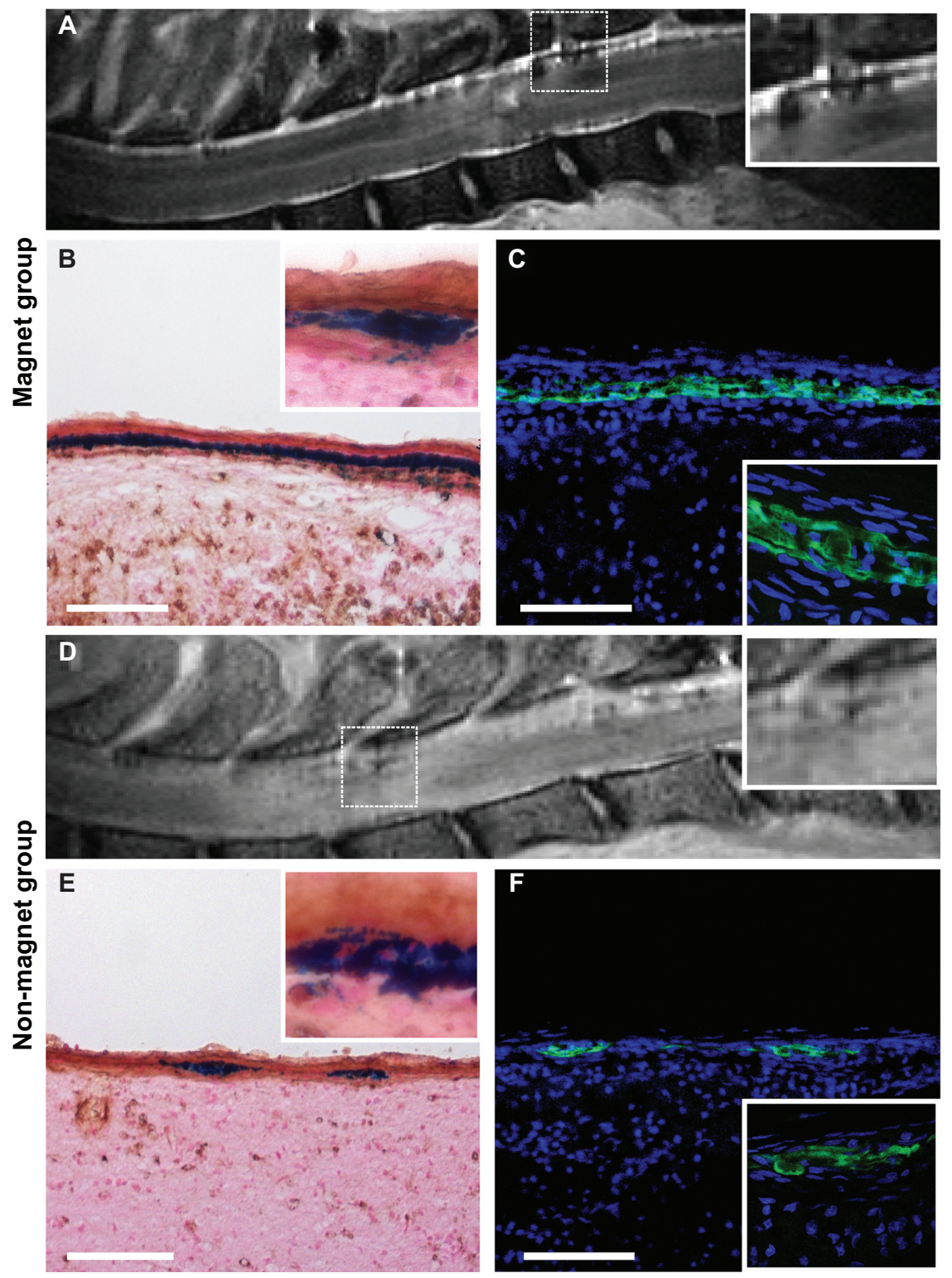

Figure 2 A strong hypointense magnetic resonance signal of SPION-labeled cells was found near the lesion area in the magnet group after intrathecal application (A) in contrast to the nonmagnet group (D). Intense Prussian blue staining (B) colocalized with the GFP signal (C) in the area under the magnet. Cell nuclei are stained with DAPI (blue) (C and F). The iron-containing cells in both groups were negative for brown ED-I staining for macrophages (B and $\mathbf{E})$ (sections counterstained with Nuclear Fast Red). A few SPIO-labeled cells were found in the lesion area in the nonmagnet group (E) colocalizing with the GFP signal (F).

Note: Scale bars $=100 \mu \mathrm{m}$.

Abbreviations: GFP, green fluorescent protein; SPION, superparamagnetic iron oxide nanoparticles; DAPI, 4',6-diamidino-2-phenylindole.

\section{Theoretical analysis of the magnetic forces acting on SPION-labeled cells from a slab-shaped permanent magnet in the spinal cord}

The magnetic force acting on a cell loaded with magnetic nanoparticles can be calculated as

$$
\begin{aligned}
& \vec{F}_{m}=\frac{V_{c} \chi}{\mu_{0}}(\vec{B} \vec{\nabla}) \vec{B}= \\
& =\frac{V_{c} \chi}{\mu_{0}}\left(B_{x} \frac{\partial}{\partial x}+B_{y} \frac{\partial}{\partial y}+B_{z} \frac{\partial}{\partial z}\right)\left(\vec{i} B_{x}+\vec{j} B_{y}+\vec{k} B_{z}\right)
\end{aligned}
$$

where $V_{c}$ is the cell volume, $\chi$ is the magnetic susceptibility of a cell relative to the cerebrospinal fluid, $\nabla$ is the operator nabla, $i, j$, and $k$ are the unit direction vectors of a Cartesian coordinate system, $\mu_{0}$ is the permeability of free space, $B=\mu_{0} H$ is the magnetic induction, and $H$ is the magnetic field strength. ${ }^{32}$ Note that the force component perpendicular (z-component) to the channel walls plays a major role in the targeted capture of cells. In Figure 3, we show the scaled magnetic force as a function of cell position on the $x$-scale, calculated for the slab-shaped magnet used in our experiments $(13 \times 7 \times 2 \mathrm{~mm})$ as described previously. ${ }^{31}$ 

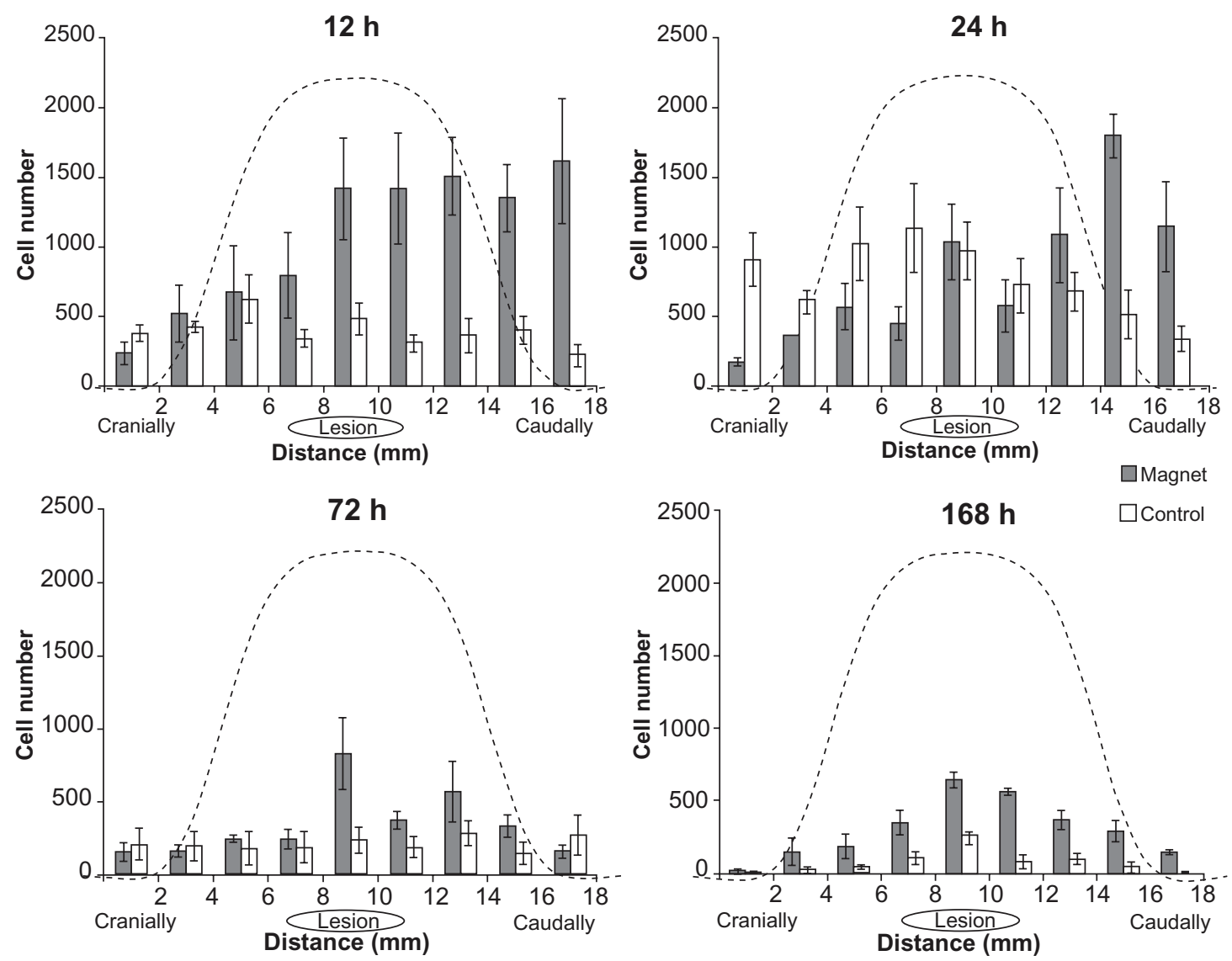

Figure 3 Distribution of cells in an $18 \mathrm{~mm}$ long segment of the injured spinal cord 12, 24, 72, and I68 hours after intrathecal injection.

Notes: The cell distribution correlates with the magnetic forces of the magnetic implant above the lesion site, which attracts and concentrates the cells near the lesion and prevents the cells from advancing more cranially. The dashed lines depict the calculated distribution of the z-component of the magnetic forces for a slab-shaped magnet at a distance of $4.5 \mathrm{~mm}$ from the magnet's surface.

Comparing the experimentally achieved stem cell distributions (Figure 3) with the force distribution, one can see their strong resemblance. The visible asymmetry in the cell distributions is obviously caused by the cells' injection into the lumbar space and their primarily unidirectional cranial migration through the cerebrospinal fluid (CSF). During stem cell targeting, the interplay between the physical distribution of the cells (governed by the magnetic forces) and their biodistribution results in the cell patterns shown in Figure 3. We suggest that by optimizing the physical (magnetic) force distribution, one can achieve a more precise and effective cell targeting near the site of injury. The main idea of the optimization method relies on the fact that magnetic cell targeting can be improved by using a magnet of special shape that produces spatially modulated stray fields. ${ }^{31,33}$

\section{Optimizing magnet geometry for focusing magnetically labeled cells}

As seen from Figure 4 (dashed curve), the calculated force distribution of a slab-shaped magnet is rather flat, and the capturing area does not fit well with the 3-4 mm-sized lesion site. For medical applications, it is often important to capture magnetic cells more locally. In such a case, the magnetic system should be optimized for narrow focusing, which could be achieved by, for example, a slab-shaped magnet with one central step. The magnetic force distribution generated with such a stepped magnet is shown in Figure 4 by the black curve (the step size is $4 \times 7 \times 2 \mathrm{~mm}$ ). Thus, adding only one step on a flat magnet significantly increases the magnetic force and allows for the more precise focusing of magnetically labeled cells to the lesion site. An additional advantage of the proposed stepped magnet is its strong horizontal force component ( $x$-component), due to which the stepped magnet attracts the magnetically labeled stem cells. Figure $5 \mathrm{~A}$ and $\mathrm{B}$ show: (i) the magnetic force distributions in the $(x, z)$-plane for flat and stepped magnets calculated analytically by using previously reported explicit expressions for the magnetic stray fields produced by a uniformly magnetized $\mathrm{slab}^{31}$ and (ii) the schematic distributions of the transplanted cells in the subarachnoid space affected by the magnetic forces. 


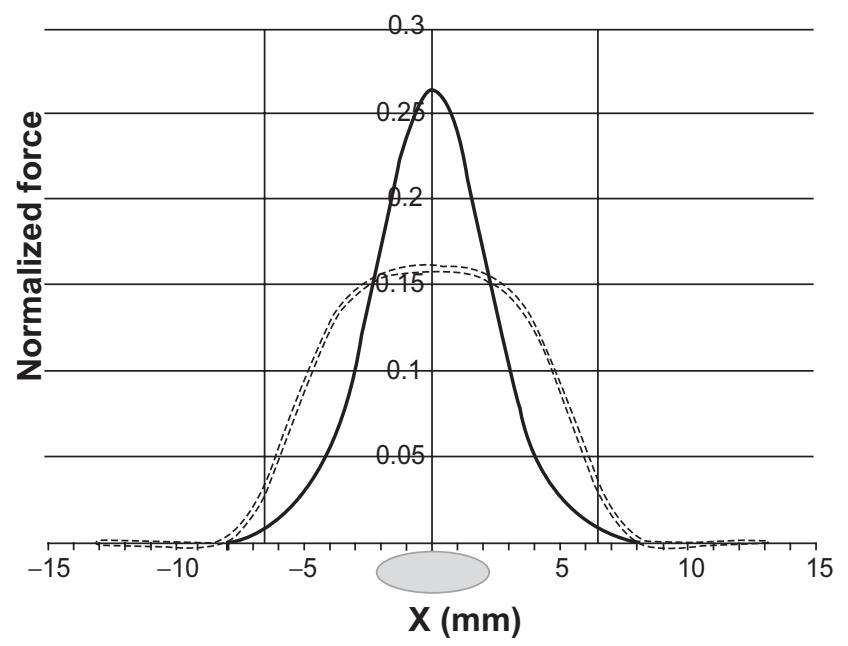

Figure 4 Scaled $z$-component of the magnetic force (normalized to $\chi \mu_{0} V_{c} M_{s}{ }^{2} / a$, where $M_{s}$ is the saturation magnetization of the magnet and $a$ is the lateral half-size of the magnet) calculated as a function of the coordinate in the cerebrospinal fluid channel for a slab-shaped magnet (dashed) and one with a central step $4 \times 7 \times 2 \mathrm{~mm}$ (black).

Note: The force was calculated for the plane $4.5 \mathrm{~mm}$ above the magnet's surface. The vertical lines indicate the boundaries of the magnet, $x_{b}= \pm 6.5 \mathrm{~mm}(13 \times 7 \times 2 \mathrm{~mm})$. The lesion site is shown as a grey ellipse at the origin of the coordinate system.

A comparison of Figure 5A and B clearly demonstrates that the use of a stepped magnet provides the planar components of the magnetic forces needed for attracting the labeled cells to the lesion site, while a flat magnet attracts cells mainly to the edge of the magnet. As seen from Figure 5B, many areas with high field gradients are located near the stepped magnet. This allows the use of such a magnet for targeted cell delivery to lesion sites of specific geometry. The magnetic field gradient value and direction, as well as the force distribution, could be tuned by changing the number of steps and their positions, as well as the step sizes and heights.

\section{Modeling cell targeting}

One of the crucial problems in stem cell targeting is its efficiency. The efficiency of magnetic targeting is limited by both physical and biological factors. Indeed, as described above, only thousands from half a million injected cells were captured near the lesion site. Hence, a description of the cell targeting by a mathematical model, taking into account the magnetic forces and the magnet's parameters, the geometry and volume of the cerebrospinal channel, and the characteristic life span of the cells would significantly advance our understanding of this dynamic process.

Let us assume that $N_{0}$ of magnetically labeled cells were injected in a channel (subarachnoid space). With time $\tau_{b i o}$, the number of cells decays on a time scale due to biological reasons: the finite life span of the cells, immunological reactions against the transplanted cells, apoptosis due to cell-to-cell interactions, possible nanoparticle toxicity, etc. On the other hand, in the channel volume, the physical process of cell capture is mainly governed by the magnetic forces acting on the stem cells loaded with SPION. This process is characterized by the characteristic time $\tau_{p h}$, which depends on the magnetic field gradient (forces), the number of SPION per cell, the magnetic moment of the nanoparticles, and the experimental geometry. The main force to be overcome by the magnetic force is the drag force of the cerebrospinal fluid, $F_{d}=6 \pi \mu R v$, where $R$ is the mean cell size, $v$ is the difference between the cell and fluid velocities, and $\mu$ is the fluid viscosity.

Assuming that magnetic forces act on cells near the magnet surface within an effective area $S$ and that the cells circulate with the cerebrospinal fluid in the subarachnoid space of an overall volume $V$, we now calculate the number of captured cells. In the whole volume, a decrease in the number of cells due to their attraction to the magnet, during time $d t$, is described as

$$
d N_{V}(t)=-\frac{N_{V}(t)<v>S d t}{V}
$$

where $N_{V}(t)$ is the remaining number of cells in the volume. Integrating Eq. 2 from 0 to $t$ and taking into account that $N_{V}(0)=N_{0}$ as well as that the number of captured cells is $N(t)=N_{0}-N_{V}(t)$, one can arrive at

$$
N(t)=N_{0}\left(1-e^{-t / \tau} p h\right)
$$

where $\tau_{p h}=V /(S<v>)$ is the characteristic time and $\langle v\rangle$ is the mean cell velocity determined by the balance of the forces: magnetic, drag and tether to the channel walls (adhesion forces). In the limiting case when $t<<\tau_{p h}$, as follows from Eq 3, the number of captured cells linearly depends on time: $N(t)=N_{0} t / \tau_{p h}$. It is also seen from Eq 3 that the capturing efficiency $N(t) / N_{0}$ and the rate $d N / d t=N_{0} /$ $\tau_{p h}=N_{0} S<v>/ V$ strongly depends on the magnetic force values (in the last expression the mean cell velocity is directly proportional to the magnetic drag force), the effective magnet area, and the channel volume.

Now we analyze the consequences of Eq. 3 and compare them with the experimental data shown in Figure 6A, where the number of trapped cells is plotted as a function of time. As seen from this figure, initially the number of captured cells linearly increases with time, then, after reaching a certain cell concentration, $N(t)$ starts to decay due to the previously mentioned biological factors. During this linear increase 

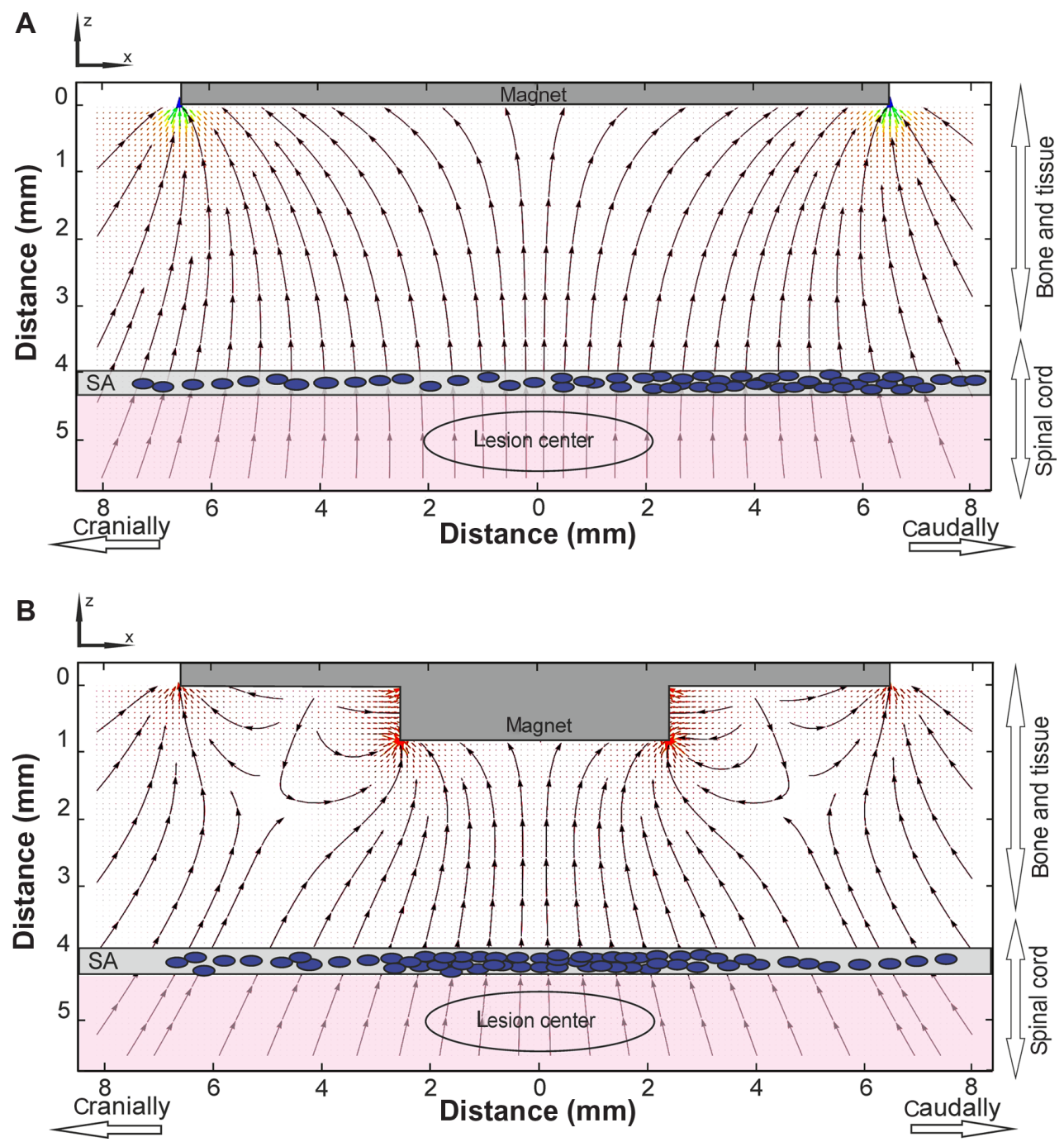

Figure 5 Magnetic force lines near the surfaces of flat $(\mathbf{A})$ and stepped $(\mathbf{B})$ magnets.

Notes: The arrow lines show the directions of forces acting on a magnetically labeled cell. The corresponding distribution of transplanted cells in the subarachnoid space of the spinal cord under both types of magnet is schematically shown by the blue dots. The areas with the highest values of the magnetic force are progressively marked in green, orange, and red.

(Figure 6A) the capturing rates are 794 and 287 cells/hour with and without the magnet, respectively. Obviously, for the same number of injected cells, the capturing rate is lower when the magnet is not present.

Our model, specified on the smallest time scale $\left(t<<\tau_{p h}\right)$, predicts the linear dependence of the captured cells on time, $N(t)=N_{0} t / \tau_{p h}$, which experimentally was observed for $t<12$ hours. From the data shown in Figure 6A, one can determine the physical characteristic time as $\tau_{p h}=12\left(N_{0} /\right.$ $N(12))=12 \cdot 0.5 \cdot 10^{6} / 9525=630$ hours $=26$ days. During this time, the majority of the injected SPION-labeled cells would theoretically be captured by the magnet in the absence of the biological factors that are responsible for cell loss. However, on the time scale $t>24$ hours (see Figure 6), the biological factors' influence exceeds that of the physical factors and causes the observed decrease in the size of the trapped cell colony. The whole process of magnetic stem cell targeting can be described by a combination of two time-dependent functions with different characteristic times: $N_{1}(t)=N_{0}\left(1-\operatorname{Exp}\left(-t / \tau_{p h}\right)\right)$ for $t<t_{0}$ and $N_{2}(t)=N_{1}\left(t_{0}\right)$ $\operatorname{Exp}\left(-\left(t-t_{0}\right) / \tau_{\text {bio }}\right)$ for $t>t_{0}$, where $t_{0}$ is the moment of time when the biological factors start to prevail over the physical ones. An exemplary plot of this dependence calculated for $\tau_{\text {bio }}=80$ hours and $t_{0}=12$ hours is shown in Figure 6B. The values of $\tau_{b i o}=80$ hours and $\tau_{p h}=630$ hours were determined by fitting the experimental data shown in Figure 6 .

It should be stressed here, that to achieve maximal efficiency in stem cell delivery in this magnetic system and experimental setup geometry, the magnet must be removed from the rat's body before the time $t_{0}$. The optimal time of 

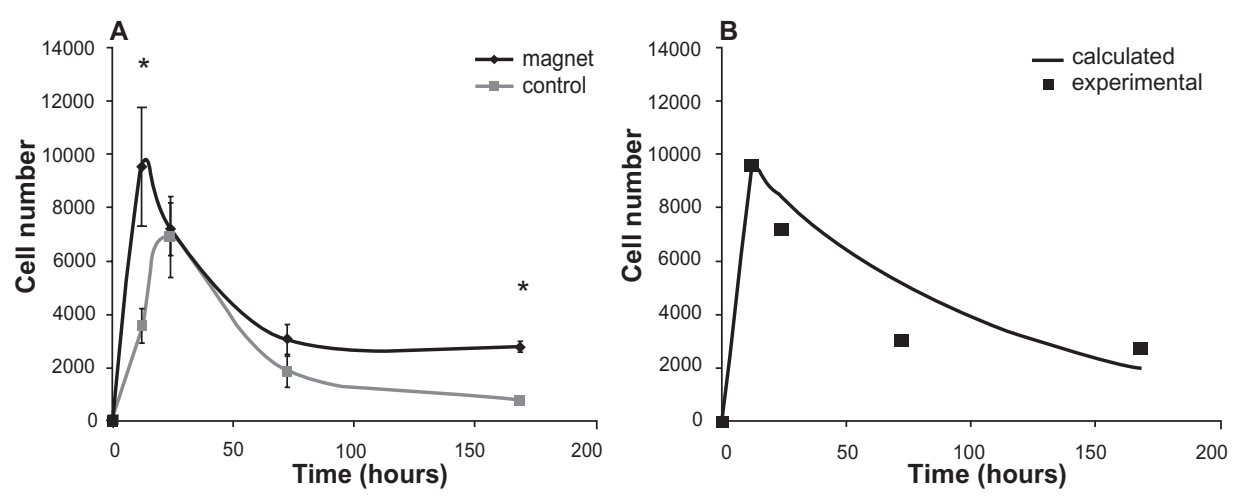

Figure 6 Total number of captured cells in the measured area with (black) and without (grey) a magnet versus time (in hours) (A). Calculated (curve) and experimental (filled squares) time dependencies of the magnetically captured cells influenced by both physical and biological factors (B).

magnet removal is determined by the interplay of the two characteristic times: $\tau_{p h}$ and $\tau_{b i o}$. Because of the fact that $\tau_{b i o}$ is hardly a controllable quantity, to reach the maximal possible efficiency one should decrease the characteristic time $\tau_{p h}=V /(S<v>)$, eg, by a proper choice of the magnet's strength, shape, and operating area. For example, as seen from the expression for the physical characteristic time $\tau_{p h}=V /(S<v>)$, the use of magnets of the smallest sizes $(L=V / S)$ with all of the magnetic parameters preserved would allow $\tau_{p h}$ to decrease and increase the capture rate. A delay in magnet removal might decrease the number of captured live cells in the lesion site because of cell death in the target area or the possible further uptake of dead cells due to cerebrospinal fluid flow. Also, cytokines released by the lesioned tissue cannot attract transplanted cells to the lesion site. By removing the magnet, one can maximize the number of live cells, which can have a therapeutic effect in the lesioned tissue. Thus, the knowledge and control of both of the characteristic times ( $\tau_{p h}$ and $\tau_{b i o}$ ) allow us to increase the targeting efficiency.

\section{Discussion}

We studied the possibility of targeting MSC labeled with SPION into the damaged spinal cord by means of magnetic targeting. In view of the fact that the number of MSC that reach the lesioned neural tissue after transplantation is considered to be low, ${ }^{34}$ especially at short time intervals postimplantation, we believe that this approach can be beneficial for improving the effectiveness of cell transplantation.

Prior to in vivo use, we tested the permanent magnetbased targeting system in vitro. In our previous study, we showed that the efficiency of cell labeling with poly-L-lysinecoated SPION ranges from $72 \%-84 \% .{ }^{35}$ After we seeded labeled cells on a Petri dish, they were attracted to the region above the magnet and concentrated in the target area.
These results are in good agreement with the findings of other authors. ${ }^{36,37}$

After the implantation of the slab-shaped magnet covered with a biocompatible silicone coating, we did not observe any pathology or inflammation around the implant, suggesting the safety of the procedure. The transplantation of labeled cells intrathecally via lumbar puncture was performed 1 week after SCI, which is considered to be within the range of the optimal therapeutic window. ${ }^{38}$ Intrathecal application has been repeatedly reported to be a clinically attractive method for cell delivery into the spinal cord because of its low invasiveness and relative simplicity. ${ }^{39}$

As shown by a hypointense MRI signal (due to the presence of SPION in the transplanted cells) and confirmed by histological staining, the transplanted cells were present in the subarachnoid space in both the magnet and nonmagnet groups and did not migrate deeply into the spinal cord tissue. Interestingly, Mothe et $\mathrm{al}^{34}$ transplanted nonlabeled MSC via lumbar puncture into the spinal cord 1 week after a compression injury and reported that the majority of transplanted cells were located in the intrathecal space distributed along the spinal cord and did not migrate into the spinal cord parenchyma. They did not observe the exclusive homing of MSC to the SCI site and suggested that after intrathecal administration, some cells might be attracted or trapped in the lesion site due to a meningeal reaction or the swelling of the spinal cord. The possible entrapment of cells in the damaged lesion tissue might explain the increase in cell numbers in the lesion observed over time in the nonmagnet control group. In contrast, other authors reported that MSC have the ability to migrate into the lesion parenchyma when transplanted intrathecally. ${ }^{40}$ This discrepancy might be explained by differences in the various types of SCI and variations in the transplant intervals, ${ }^{34}$ since the migration of cells from the CSF to the lesion might vary in different types of SCI. 
The cerebrospinal fluid circulation within the subarachnoid space functions as a closed hydraulic system protecting the central nervous system. ${ }^{41}$ It must be noted that the circulation of the CSF plays an important role in cell transport after intrathecal transplantation. There are several models of CSF circulation suggested in the literature; ${ }^{42-44}$ however, the flow dynamics of the CSF are still not fully understood. CSF circulation might also vary between pathologic and normal conditions.

Based on our results, we suggest that the magnet should be removed in order to efficiently target the cells to the lesion site. It must be taken into account that without the removal of the magnet, the magnet might even prevent the cells from migrating deeply into the lesion site. Recently, Sasaki et $\mathrm{al}^{45}$ reported a significant effect of SPION-labeled MSC magnetically targeted via the CSF to a spinal cord lesion on the behavioral outcome of rats with SCI. They observed the transplanted cells on the surface of the spinal cord in the subarachnoid space; the cells did not migrate into the spinal cord tissue. However, they did not report a detailed evaluation of their magnetic system nor whether they removed the magnet or not. Experimental validation of an increase in capturing efficiency caused by magnet removal at the proper time will be presented in a forthcoming paper.

We have shown that in the lesioned area of the spinal cord, there was a significant difference in cell distribution between the magnet and nonmagnet groups. The cells circulating in the subarachnoid space were gathered under the magnetic implant. This correlates well with the study of Nishida et al. ${ }^{26}$ These authors measured the area of GFPpositive cells in the healthy spinal cord under a magnetic implant (5 $\mathrm{mm}$ in diameter, $3 \mathrm{~mm}$ in height, $350 \mathrm{mT}$ ) after the administration of SPION-labeled cells via lumbar puncture and reported a higher concentration of cells in the subarachnoid space below the magnetic implant one day after intrathecal transplantation. In contrast to Nishida et al, we directly counted the number of implanted cells instead of measuring the GFP-positive area, a more accurate approach to determining the number of cells and the cell distribution in the lesion site. As we have shown, the number of cells that can be concentrated near the target area by using a simple slab-shaped permanent magnet was significantly higher in comparison to the non-magnet group. These findings agree with theoretical calculations of the magnetic properties of such a magnetic implant. However, the distribution of the (z) component of the magnetic force of a slab-shaped magnet is flat and does not precisely focus the cells at the lesion site. Moreover, due to the weak planar (x) component of the magnetic force, the cells are not effectively attracted with such a magnet.

Periodically magnetized structures producing nonuniform magnetic field spatial distributions for magnetic targeting applications were previously studied analytically as well as by simulations by Häfeli et al ${ }^{46}$ However, to the best of our knowledge, the theoretical concepts of magnetic targeting in the spinal cord are poorly discussed in the literature. In order to predict the behavior of a magnetic targeting system, we introduced a mathematical model in which the process of cell targeting is characterized by the specific times $\tau_{p h}$ and $\tau_{b i o}$.

One of the crucial factors influencing the $\tau_{\text {bio }}$ of a transplanted cell is the dose and type of iron oxide nanoparticle used for cell labeling. As we recently showed, SPION might cause oxidative damage to biological macromolecules as well as DNA damage, which might in turn influence the biological behavior of the cells and $\tau_{b i o}{ }^{47}$ Various types of SPION with different coatings are under development; however, the most appropriate type and dose of nanoparticles for cell labeling and magnetic targeting remain to be determined.

Considering the limitations of the above-mentioned magnetic system based on a simple slab-shaped magnet, we suggested an improved tunable magnetic system allowing for the precise focusing of magnetically labeled cells to a target in the spinal cord that will be experimentally tested in future experiments.

\section{Conclusion}

In conclusion, the targeting of SPION-labeled MSC into the injured rat spinal cord was achieved with the use of an implanted slab-shaped permanent magnet. We showed how the magnetic implant affects the distribution and kinetics of the transplanted cells in the spinal cord after intrathecal implantation. The magnetic system based on a commercially available magnet appears to be functional despite being limited by both physical and biological factors. These limitations are discussed, and the improvement of the magnetic targeting system by tuning the magnetic force distribution is suggested. The proposed magnetic delivery system can be attractive for clinical application development.

\section{Acknowledgment}

This work was supported by the Grant Agency of the Czech Republic (P304/12/1370, P304/11/0731, P304/11/0189, P304/11/0653) and the Grant Agency of the Academy of Sciences of the Czech Republic (AVOZ50390703, IAA500390902, AV0Z10100522). The authors thank James Dutt for his critical reading of the manuscript. 


\section{Disclosure}

The authors report no conflicts of interest in this work.

\section{References}

1. Kubinova S, Sykova E. Biomaterials combined with cell therapy for treatment of spinal cord injury. Regen Med. 2012;7(2):207-224.

2. Talac R, Friedman JA, Moore MJ, et al. Animal models of spinal cord injury for evaluation of tissue engineering treatment strategies. Biomaterials. 2004;25(9):1505-1510.

3. Sykova E, Homola A, Mazanec R, et al. Autologous bone marrow transplantation in patients with subacute and chronic spinal cord injury. Cell Transplant. 2006;15(8-9):675-687.

4. Arboleda D, Forostyak S, Jendelova P, et al. Transplantation of predifferentiated adipose-derived stromal cells for the treatment of spinal cord injury. Cell Mol Neurobiol. 2011;31(7):1113-1122.

5. Kim J, Kim IS, Cho TH, et al. Bone regeneration using hyaluronic acid-based hydrogel with bone morphogenic protein-2 and human mesenchymal stem cells. Biomaterials. 2007;28(10):1830-1837.

6. Fan $\mathrm{H}, \mathrm{Hu} \mathrm{Y}, \mathrm{Zhang} \mathrm{C}$, et al. Cartilage regeneration using mesenchymal stem cells and a PLGA-gelatin/chondroitin/hyaluronate hybrid scaffold Biomaterials. 2006;27(26):4573-4580.

7. Gaebel R, Ma N, Liu J, et al. Patterning human stem cells and endothelial cells with laser printing for cardiac regeneration. Biomaterials. 2011; 32(35):9218-9230.

8. Borlongan CV, Glover LE, Tajiri N, Kaneko Y, Freeman TB. The great migration of bone marrow-derived stem cells toward the ischemic brain: therapeutic implications for stroke and other neurological disorders. Prog Neurobiol. 2011;95(2):213-228.

9. Forostyak S, Jendelova P, Kapcalova M, Arboleda D, Sykova E. Mesenchymal stromal cells prolong the lifespan in a rat model of amyotrophic lateral sclerosis. Cytotherapy. 2011;13(9):1036-1046.

10. Kubinova S, Sykova E. Nanotechnology for treatment of stroke and spinal cord injury. Nanomedicine (Lond). 2010;5(1):99-108.

11. Urdzikova L, Jendelova P, Glogarova K, Burian M, Hajek M, Sykova E. Transplantation of bone marrow stem cells as well as mobilization by granulocyte-colony stimulating factor promotes recovery after spinal cord injury in rats. J Neurotrauma. 2006;23(9):1379-1391.

12. Osaka M, Honmou O, Murakami T, et al. Intravenous administration of mesenchymal stem cells derived from bone marrow after contusive spinal cord injury improves functional outcome. Brain Res. 2010;1343: 226-235.

13. Chopp M, Li Y. Treatment of neural injury with marrow stromal cells. Lancet Neurol. 2002;1(2):92-100.

14. Akiyama Y, Radtke C, Honmou O, Kocsis JD. Remyelination of the spinal cord following intravenous delivery of bone marrow cells. Glia. 2002;39(3):229-236

15. Chen X, Katakowski M, Li Y, et al. Human bone marrow stromal cell cultures conditioned by traumatic brain tissue extracts: growth factor production. J Neurosci Res. 2002;69(5):687-691.

16. Aggarwal S, Pittenger MF. Human mesenchymal stem cells modulate allogeneic immune cell responses. Blood. 15 2005;105(4):1815-1822.

17. Crigler L, Robey RC, Asawachaicharn A, Gaupp D, Phinney DG. Human mesenchymal stem cell subpopulations express a variety of neuro-regulatory molecules and promote neuronal cell survival and neuritogenesis. Exp Neurol. 2006;198(1):54-64.

18. Wilkins A, Kemp K, Ginty M, Hares K, Mallam E, Scolding N. Human bone marrow-derived mesenchymal stem cells secrete brain-derived neurotrophic factor which promotes neuronal survival in vitro. Stem Cell Res. 2009;3:63-70.

19. Paul C, Samdani AF, Betz RR, Fischer I, Neuhuber B. Grafting of human bone marrow stromal cells into spinal cord injury: a comparison of delivery methods. Spine (Phila Pa 1976). 2009:328-334.

20. Jendelova P, Herynek V, Urdzikova L, et al. Magnetic resonance tracking of transplanted bone marrow and embryonic stem cells labeled by iron oxide nanoparticles in rat brain and spinal cord. J Neurosci Res. 2004; $76(2): 232-243$
21. Sykova E, Jendelova P. Magnetic resonance tracking of implanted adult and embryonic stem cells in injured brain and spinal cord. Ann N Y Acad Sci. 2005;1049:146-160.

22. Huang Z, Pei N, Wang Y, et al. Deep magnetic capture of magnetically loaded cells for spatially targeted therapeutics. Biomaterials. 2010; 31(8):2130-2140.

23. Wilhelm C, Bal L, Smirnov P, et al. Magnetic control of vascular network formation with magnetically labeled endothelial progenitor cells. Biomaterials. 2007;28(26):3797-3806.

24. Arbab AS, Jordan EK, Wilson LB, Yocum GT, Lewis BK, Frank JA. In vivo trafficking and targeted delivery of magnetically labeled stem cells. Hum Gene Ther. 2004;15(4):351-360.

25. Song M, Kim YJ, Kim YH, Roh J, Kim SU, Yoon BW. Using a neodymium magnet to target delivery of ferumoxide-labeled human neural stem cells in a rat model of focal cerebral ischemia. Hum Gene Ther. 2010;21(5):603-610.

26. Nishida K, Tanaka N, Nakanishi K, et al. Magnetic targeting of bone marrow stromal cells into spinal cord: through cerebrospinal fluid. Neuroreport. 21 2006;17(12):1269-1272.

27. Dominici M, Le Blanc K, Mueller I, et al. Minimal criteria for defining multipotent mesenchymal stromal cells. The International Society for Cellular Therapy position statement. Cytotherapy. 2006;8:315-317.

28. Babic M, Horak D, Trchova M, et al. Poly(L-lysine)-modified iron oxide nanoparticles for stem cell labeling. Bioconjug Chem. 2008;19(3): $740-750$.

29. De la Calle JL, Paino CL. A procedure for direct lumbar puncture in rats. Brain Res Bull. 2002;59(3):245-250.

30. Mothe AJ, Tator CH. Transplanted neural stem/progenitor cells generate myelinating oligodendrocytes and Schwann cells in spinal cord demyelination and dysmyelination. Exp Neurol. 2008;213(1): 176-190.

31. Zablotskii V, Pastor JM, Larumbe S, et al. High-field gradient permanent micromagnets for targeted drug delivery with magnetic nanoparticles. AIP Conf. Proc. 2010;1311:152-157.

32. Pankhurst QA, Connolly J, Jones SK, Dobson J. Applications of magnetic nanoparticles in biomedicine. J Phys D Appl Phys. 2003; 36(13):R167-R181

33. Zablotskii V, Lunov O, Dejneka A, et al. Nanomechanics of magnetically driven cellular endocytosis. Appl Phys Lett. 2011;99(18): 183701-183703.

34. Mothe AJ, Bozkurt G, Catapano J, et al. Intrathecal transplantation of stem cells by lumbar puncture for thoracic spinal cord injury in the rat. Spinal Cord. 2011;49(9):967-973.

35. Horak D, Babic M, Jendelova P, et al. Effect of different magnetic nanoparticle coatings on the efficiency of stem cell labeling. J Magn Magn Mater. 2009;321(10):1539-1547.

36. Cheng K, Li TS, Malliaras K, Davis DR, Zhang Y, Marban E. Magnetic targeting enhances engraftment and functional benefit of iron-labeled cardiosphere-derived cells in myocardial infarction. Circ Res. 2010; 106(10):1570-1581.

37. Schafer R, Bantleon R, Kehlbach R, et al. Functional investigations on human mesenchymal stem cells exposed to magnetic fields and labeled with clinically approved iron nanoparticles. BMC Cell Biol. 2010;11:22.

38. Sykova E, Jendelova P, Urdzikova L, Lesny P, Hejcl A. Bone marrow stem cells and polymer hydrogels-two strategies for spinal cord injury repair. Cell Mol Neurobiol. 2006;26(7-8):1113-1129.

39. Bakshi A, Hunter C, Swanger S, Lepore A, Fischer I. Minimally invasive delivery of stem cells for spinal cord injury: advantages of the lumbar puncture technique. J Neurosurg Spine. 2004;1(3):330-337.

40. Satake K, Lou J, Lenke LG. Migration of mesenchymal stem cells through cerebrospinal fluid into injured spinal cord tissue. Spine (Phila Pa 1976). 2004;29(18):1971-1979.

41. Pardridge WM. Drug transport in brain via the cerebrospinal fluid. Fluids Barriers CNS. 2011;8:7.

42. Di Chiro G. Observations on the circulation of the cerebrospinal fluid. Acta Radiol Diagn (Stockh). 1966;5:988-1002. 
43. Greitz D, Hannerz J. A proposed model of cerebrospinal fluid circulation: observations with radionuclide cisternography. AJNR Am J Neuroradiol. 1996;17(3):431-438.

44. Linninger AA, Xenos M, Sweetman B, Ponkshe S, Guo X, Penn R. A mathematical model of blood, cerebrospinal fluid and brain dynamics. J Math Biol. 2009;59(6):729-759.

45. Sasaki H, Tanaka N, Nakanishi K, et al. Therapeutic effects with magnetic targeting of bone marrow stromal cells in a rat spinal cord injury model. Spine (Phila Pa 1976). 2011;36(12):933-938.
46. Häfeli UO, Gilmour K, Zhou A, Lee S, Hayden ME. Modeling of magnetic bandages for drug targeting: Button vs Halbach arrays. J Magn Magn Mater. 2007;311(1):323-329.

47. Novotna B, Jendelova P, Kapcalova M, et al. Oxidative damage to biological macromolecules in human bone marrow mesenchymal stromal cells labeled with various types of iron oxide nanoparticles. Toxicol Lett. 2012;210(1):53-63.

\section{Publish your work in this journal}

The International Journal of Nanomedicine is an international, peerreviewed journal focusing on the application of nanotechnology in diagnostics, therapeutics, and drug delivery systems throughout the biomedical field. This journal is indexed on PubMed Central, MedLine, CAS, SciSearch $\AA$, Current Contents ${ }^{\circledR} /$ Clinical Medicine,
Journal Citation Reports/Science Edition, EMBase, Scopus and the Elsevier Bibliographic databases. The manuscript management system is completely online and includes a very quick and fair peer-review system, which is all easy to use. Visit http://www.dovepress.com/ testimonials.php to read real quotes from published authors. 